\title{
Blue Light Exposure In The Morning and Low-Dose Aripiprazole Administration at Night Combined to Effectively Treat Wake-Up Difficulty due to Prolonged Sleep Time
}

\author{
Tetsuro Hoshino, Toshiaki Shiomi*, Noriyuki Konishi, Maiko Suda, Yoko Haseda, Mamiko Mano, Atsuhiko Nomura, \\ Reiko Hori and Ryujiro Sasanabe
}

Department of Sleep Medicine and Sleep Disorders Center, Aichi, Japan

Received: 네: December 15, 2018; Published: 制: January 03, 2019

*Corresponding author: Toshiaki Shiomi, Professor, Aichi Medical University School of Medicine, Director, Sleep Disorders Center, Aichi Medical University Hospital 1-1 Yazako Karimata, Nagakute, Aichi Japan

Abstract

School non-attendance due to difficulty waking up is a social concern among Japanese students. Many factors result in wake-up difficulty, and it is often difficult to identify the underlying cause at the first visit to a clinic or hospital. We, therefore assessed the effect of combination treatment with morning blue light exposure, low-dose aripiprazole (dopamine D2 and/or D3 receptor partial agonist), and sleep hygiene education in a 15-year-old patient with a 2-year history of wake-up difficulty. In this study, we demonstrate a beneficial effect with a good response of this combination treatment.

Keywords: School Non-Attendance; Wake-Up Difficulty; Circadian Rhythm Sleep-Wake Disorder; Aripiprazole; Blue Light Therapy; Sleep Hygiene Education

\section{Introduction}

School attendance is essential for acquisition of both academic and social-emotional functions during development. In Japan, there is an increasing number of adolescents with school non-attendance, and this is recognized as a significant social concern. Although several factors can be reasons for school non-attendance caused by difficulty waking up, lack of synchronization of sleep-wake rhythms to the local time caused by chronic insufficient sleep time, delayed sleep phase syndrome (DSPS), or prolonged total sleep time are considered the most common in these students. DSPS is a circadian rhythm sleep-wake disorder (CRSWD) defined by a persistent delay in sleep onset and wake times, with excessive early day sleepiness, and extreme and prolonged difficulty awaking up with morning confusion. Additionally, prolonged sleep time is an aspect of DSPS. It is necessary to distinguish this from patterns of DSPS in patients from "normal" delayed sleep patterns commonly seen in adolescents, who prefer delayed sleep schedules as a pubertal transition without functional impairment or social distress, but it is difficult to differentiate these at the first visit to a clinic or hospital. To treat these patients, it is, therefore, advisable to carry out a combination treatment.
For healthy sleep, it is important to regulate the circadian rhythm. Typically, morning bright light therapy with a scheduled rise time is used for CRSWD treatment [1]. Recently, low-dose aripiprazole (APZ), which is a second-generation antipsychotic drug acting as partial agonist of dopamine D2 and/or D3 receptor, was reported to advance sleep rhythm and reduce prolonged sleep time in patients with DSPS [2]. Finally, sleep hygiene education is essential to make these students aware of the effect of chronic insufficient sleep. Adolescents have been determined to require approximately $9.25 \mathrm{~h}$ sleep per night, and many adolescents obtain less sleep than they actually need. Here, we report the case of a 15 -year-old student with school non-attendance and difficulty waking. For his treatment, we combined the above-mentioned approaches by administering treatment with morning bright light therapy using blue light for home use (Philips goLITE BLU HF3331) (Figure 1), low-dose APZ (3mg/day), and sleep hygiene education. Our results indicate that the student responded well to this combination treatment with a stabilization of sleep time soon after initiation of the intervention. 


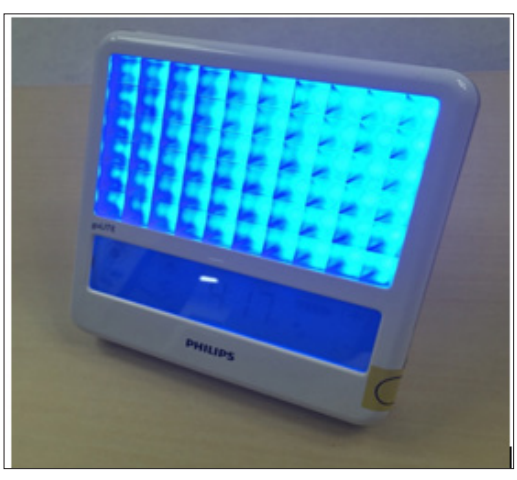

Figure 1: Philips goLITE BLU HF3331 for home use. The goLITE BLU is commercially available and has peak transmission at $470 \mathrm{~nm}$, intensity at the cornea $300 \mathrm{lux}$, 2306 m-lux.

\section{Case Presentation}

A 15-year-old otherwise healthy junior high school student presented to us with a two-year history of school non-attendance due to wake-up difficulty. Before the problem began, he had been sleeping 12 hours and attending school without any problems. From age 13, he had started excessively using the internet on his smartphone and as shown in (Figure 2), had started to have difficulty falling asleep and waking up at the desired time to prepare for school, resulting in school non-attendance. Before he came to our hospital, melatonergic administration (Ramelteon 4mg/ day) had been attempted, but there had been no improvement. On his first visit to our hospital, Ramelteon administration was discontinued, and we initiated morning blue light therapy with administration of APZ ( $3 \mathrm{mg} /$ day) at $7 \mathrm{pm}$. Additionally, the patient was instructed to go to bed as 9pm. Overnight polysomnography (PSG) was performed on day 17 after treatment initiation, where we excluded sleep-related breathing disorders. We were also able to exclude hypersomnia by multiple sleep latency test consisting of 5 naps conducted on the day after PSG.

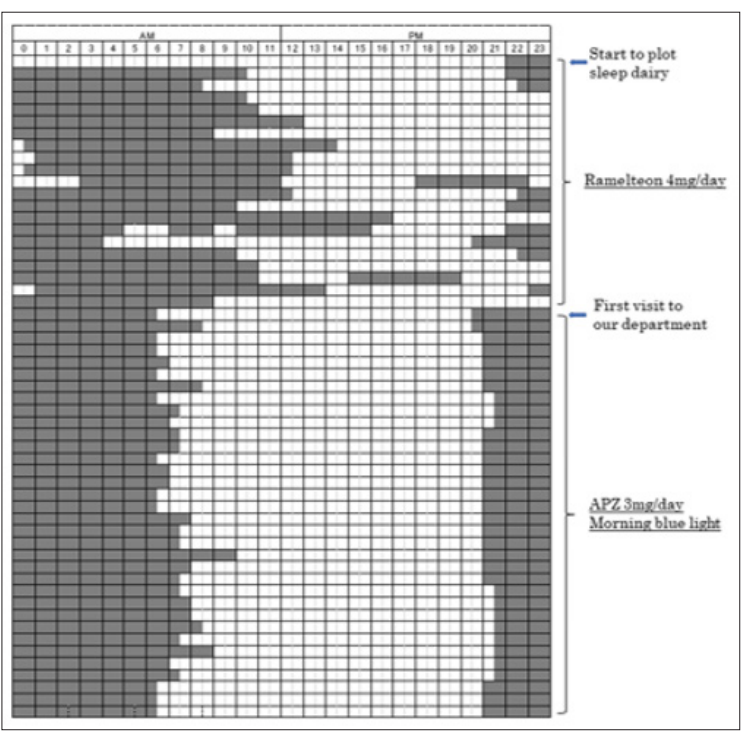

Figure 2: Sleep diary before and after first visit at our hospital. The left and right ends are $0 \mathrm{am}$. Sleep time during the 24 hours is indicated by black bars. APZ, aripiprazole.
Our treatments resulted in a significantly earlier mean sleep onset and offset time, from 24.0 (22.3-24.3) PM to 21.0 (21.0-21.5) PM, and 11.0 (9.5-12.8) AM to 7.5 (6.5-8.0) AM, respectively. The mean total sleep time was significantly reduced from 12.5 (10.8$13.3)$ to 10.0 (9.5-10.5) hours. Soon after intervention, advances in sleep phase occurred (Figure 2). Additionally, daytime sleepiness as measured by Epworth Sleepiness Score (ESS), and depression as measured by using a Self-Rating Depression Scale (SDS), improved from 12 to 4 points in ESS and 42 to 38 points in SDS, respectively. Health-related quality of life as measured by SF-36 score (in physical function, vitality, social function, and mental health) also improved for each item, and the patient was able to attend school again.

\section{Discussion}

This is the first case report demonstrating the effectiveness of a combination of morning blue light, low-dose aripiprazole, and sleep hygiene education to treat a student with school nonattendance due to wake-up difficulty. Although predominantly broad spectrum white light has previously been used for bright light therapy, researchers recently reported that the circadian rhythm is more responsive to shorter wavelength light (blue to green) than longer wavelength light (yellow to red) [3]. Moreover, blue light influences wakefulness and arousal; it decreases delta-theta range power density $(0.5-5.5 \mathrm{~Hz})$ and increases high-alpha range power density $(9.5-10.0 \mathrm{~Hz})$ on the EEG, as well as elevating core body temperature and heart rate $[4,5]$. We therefore hypothesized that morning blue light exposure could be an effective treatment for these students. Experimental models showed that dopamine regulates the circadian rhythm [6], and several case reports have demonstrated that dopaminergic upregulation using low-dose APZ, a partial agonist of dopamine D2 and/or D3 receptor, advanced the sleep-wake phase and reduced total sleep time $[2,7,8]$.

These results indicated that low-dose APZ could prove effective for the patients not only with CRSWD, but also prolonged sleep duration. Therefore, we considered that low-dose APZ could be an effective adjunctive treatment for students experiencing wakeup difficulty. Although a delay in the sleep-wake cycle is normal during adolescence, an earlier sleep schedule is necessary in order to obtain sufficient sleep and wake up at a socially appropriate time [9]. In addition, a previous study demonstrated that an earlier sleep schedule can advance circadian rhythms [10]. Therefore, we provided sleep hygiene education for all students who came to our hospital in order for them to understand the positive effect of earlier sleep. In conclusion, while there are many factors affecting school non-attendance due to wake-up difficulty in students, we propose that this combination intervention could provide effective treatment, although further research is required to establish a protocol of treatment.

\section{References}

1. Rosenthal NE, Joseph Vanderpool JR, Levendosky AA, Johnston SH, Allen R, et al. (1990) Phase-shifting effects of bright morning light as treatment for delayed sleep phase syndrome. Sleep 13: 354-361.

2. Omori Y, Kanbayashi T, Sagawa Y, Imanishi A, Tsutsui K, et al. (2018) Low dose aripiprazole advanced sleep rhythm and reduced nocturnal sleep time in the patients with delayed sleep phase syndrome: an openlabeled clinical observation. Neuropsychiatr Dis Treat 14: 1281-1286. 
3. Wright HR, Lack LC, Kennaway DJ (2004) Differential effects of light wavelength in phase advancing the melatonin rhythm. J Pineal Res 36 140-144.

4. Lockley SW, Evans EE, Scheer FA, Brainard GC, Czeisler CA, et al. (2006) Short wavelength sensitivity for the direct effects of light on alertness, vigilance, and waking electroencephalogram in humans. Sleep 29: 161168.

5. Cajochen C, Munch M, Kobialka S, Kräuchi K, Steiner R, et al. (2005) High sensitivity of human melatonin, alertness, thermoregulation, and heart rate to short wavelength light. J Clin Endocrinol Metab 90: 1311-1316.

6. Fifel K, Cooper HM (2014) Loss of dopamine disrupts circadian rhythms in a mouse model of Parkinson ss disease. Neurobiol Dis 71: 359-369.

ISSN: 2574-1241

DOI: 10.26717/BJSTR.2019.12.002299

Tetsuro Hoshino. Biomed J Sci \& Tech Res

(c) (P) This work is licensed under Creative

Submission Link: https://biomedres.us/submit-manuscript.php
7. Takaki M, Ujike H (2014) Aripiprazole is effective for treatment of delayed sleep phase syndrome. Clin Neuropharmacol 37: 123-124.

8. Matsui K, Takaesu Y, Inoue T, Inada K, Nishimura K, et al. (2017) Effect of aripiprazole on non-24-hour sleep-wake rhythm disorder comorbid with major depressive disorder: a case report. Neuropsychiatr Dis Treat 13: $1367-1371$.

9. Gradisar M, Gardner G, Dohnt H (2011) Recent worldwide sleep patterns and problem during adolescence. a review and meta-analysis of age, region, and sleep. Sleep Med 12: 110-118.

10. Sharkey KM, Carskadon MA, Fiquerio MG, Zhu Y, Rea MS, et al. (2011) Effects of an advanced sleep schedule and morning short wavelength light exposure on circadian phase in young adults with late sleep schedules. Sleep 12: 685-692.

\begin{tabular}{ll} 
BIOMEDICAL & Assets of Publishing with us \\
RESEARCHES & - Global archiving of articles \\
& - Immediate, unrestricted online access \\
& - Rigorous Peer Review Process \\
\hline hittps://biomedres.us/
\end{tabular}

